\title{
ACETATO DE CLORMADINONA EN MICRODOSIS COMO ANTICONCEPTIVO*
}

\author{
Dr. Jaime Botero 1 \\ Dr. Fabio Sánchez ${ }^{1}$ \\ Dr. Guillermo Henao ${ }^{1}$ \\ Dr. Luis F. Duque ${ }^{2}$
}

Martínez-Manautou y colaboradores (1) fueron los primeros en usar con propósito anticonceptivo un método de administración continua de 0.5 mg. de acetato de clormadinona, que se esperaba no inhibiría la ovulación y por consiguiente no alteraría el ritmo menstrual normal de las usuarias. Sus investigaciones (2) han demostrado que la tasa de embarazos es de 1,5 a 2,1 por cien mujeres-año de exposición y que los ciclos menstruales experimentan una variación poco notoria, ya que en un mes dado entre 60 y el $74 \%$ de las mujeres tienen ciclos con duración de 25 a 35 días; en un tercio no ocurre ninguna irregularidad, y aunque en un $20,5 \%$ se presenta hemorragia por rompimiento en el primero de ellos, ésta disminuye hasta un $11,9 \%$ hacia el quinto. Las reacciones secundarias son mínimas y la aceptabilidad muy buena.

Un trabajo similar al anterior fue realizado por Zañartu y colaboradores (3), quienes encontraron una tasa de embarazos de 8,4 por cien mujeres-año de exposición, alguna irregularidad de las menstruaciones en un $29 \%$ de los casos y cambios en la periodicidad de los ciclos en un $38 \%$.
Hasta el presente no se conocen los resultados del empleo de acetato de clormadinona con propósito anticonceptivo en las dosis de 0,6, 0,75 y $0,85 \mathrm{mg}$.

\section{Objetivos}

Nos proponemos en el presente estudio:

1. Conocer el efecto anticonceptivo del acetato de clormadinona en las dosis de 0,5, 0,6, 0,75 y 0,85 mg. administrado diariamente en forma continua.

2. Conocer los efectos adversos de tipo digestivo, psicológico, sobre la líbido, etc.

3. Estudiar las menstruaciones en cuanto a su periodicidad, cantidad y duración.

4. Determinar la aceptabilidad del método.

* Estudio patrocinado por el grant No 22 de la E. R. Squibb \& Sons International. Presentado al VI Congreso Mundial de Ginecología y Obstetricia. The Americana, New York, N. Y. Abril 12-18, 1970.

1 Del Departamento de Ginecología y Obstetricia, Facultad de Medicina, Universidad de Antioquia.

2 De la Escuela de Salud Pública, de la Universidad de Antioquia. 


\section{Material y Métodos}

El presente es un estudio de tipo clínico. Las mujeres que ingresaron a él pertenecen a las que asisten a las consultas de planificación familiar y de Post-parto del Hospital Universitario San Vicente y de la Clínica de Maternidad Luz Castro, de Medellín, Colombia, que eligen este método entre los que se enseñan cuando acuden en busca de orientación para planificar la familia. El método de estudio ha sido la entrevista personal, la cual se realiza mensualmente cuando la mujer va a la consulta por su dosis de pastillas; en ella se pregunta sobre efecto anticonceptivo, efectos adversos, tipo de menstruaciones y aceptabilidad del método. Las preguntas son hechas por la visitadora social y el médico asignado al programa, quien hace también exámenes clínicos y realiza procedimientos terapéuticos cuando están indicados. La aceptabilidad del método se mide de manera indirecta mediante el análisis de las causas de retiro de las usuarias, así como por la omisión de pastillas.

\section{Resultados}

Han ingresado a este estudio un total de 408 mujeres, cuya distribución de acuerdo a la dosis que están to- mando y al total acumulado de meses de uso aperecen en el Cuadro 1. En él se observa que los tres últimos grupos alcanzan cada uno una cifra de meses de uso que está alrededor de los 400. El grupo que está tomando la dosis de 0,5 mg. es el de más reciente formación y por ello en él han ocurrido menos meses de uso. Solamente se ha presentado un embarazo, en el grupo de usuarias que está tomando la dosis de 0,6 mg., mientras ingería las pastillas en forma regular.

\section{CUADRO 1}

NUMERO DE MUJERES QUE ESTAN TOMANDO DISTINTAS DOSIS DE ACETATO DE CLORMADINONA SEGUN MESES DE USO

\begin{tabular}{ccc}
\hline Dosis en $\mathbf{~ m g .}$ & $\mathbf{N}^{\mathbf{0}}$ de mujeres & Meses de uso \\
\hline 0,50 & 131 & 232 \\
0,60 & 102 & 395 \\
0,75 & 87 & 408 \\
0,85 & 88 & 418 \\
TOTAL & 408 & 1.453 \\
\hline
\end{tabular}

En el Cuadro 2 se aprecia la duración del ciclo menstrual en días. El número de meses de uso es de solo 656, pues hay un grupo de usuarias los cuatro grupos, alrededor de una tercera parte de los ciclos tiene una duración menor de 20 días. En el grupo de 0,5 mg. esa proporción lle-

\section{CUADRO 2}

DISTRIBUCION PORCENTUAL DE LA DURACION DEL SANGRADO MENSTRUAL MIENTRAS SE ADMINISTRA LA DROGA

\begin{tabular}{lccccc}
\hline Distribución del Ciclo & \multicolumn{5}{c}{ Dosis en Mg. } \\
\hline en días & 0,50 & 0,60 & 0,75 & 0,85 & Total \\
Menos de 20 & 52,0 & 32,3 & 35,4 & 38,4 & 240 \\
$21-27$ & 18,7 & 26,1 & 25,1 & 27,3 & 168 \\
$28-34$ & 8,3 & 22 & 20,1 & 15,5 & 124 \\
$35-60$ & 20,8 & 18,2 & 19,2 & 18,4 & 124 \\
Total porcentual & 99,8 & 98,6 & 99,8 & 99,6 & 656 \\
Total de Ciclos & 48 & 195 & 223 & 190 & \\
\hline
\end{tabular}


ga a la mitad de los ciclos, pero es preciso recordar que éste tiene el menor número de meses acumulado. Los ciclos de 35 y más días se presentan en un $20 \%$ del total aproximadamente, en todos los grupos. Los ciclos entre 21 y 34 días, que se consideran los más normales, ocurren en un $45 \%$ de los casos.
En el Cuadro 3 se aprecia la duración del sangrado menstrual. En la mayoría de los casos es menor de 7 días; se observan variaciones según la dosis, pues mientras que con la de $0,5 \mathrm{mg}$. el $90 \%$ de las mujeres tiene menstruaciones de menos de 7 días, con la de 0,85 ese porcentaje es solamente del 62 .

\section{CUADRO 3}

DISTRIBUCION PORCENTUAL DE LA DURACION DEL CICLO MENSTRUAL MIENTRAS SE ADMINISTRA LA DROGA

\begin{tabular}{lccccc}
\hline $\begin{array}{l}\text { Duración del sangrado } \\
\text { en días }\end{array}$ & $\begin{array}{l}\text { Total de } \\
\text { pacientes }\end{array}$ & \multicolumn{2}{c}{ Dosis en $\mathbf{M g}}$. \\
\hline menos de 7 & 502 & 100,0 & 100,0 & 100,0 & 100,0 \\
8 y más & 154 & 0,50 & 0,60 & 0,75 & 0,85 \\
Total \% & & 89,5 & 77,4 & 77,1 & 62,1 \\
Número de Cíclos & 656 & 10,5 & 22,6 & 22,9 & 37,9 \\
& & 48 & 195 & 223 & 190 \\
\hline
\end{tabular}

Ocurrió un total de 284 metrorragias o casos de sangrado intermenstrual en 135 mujeres. De esos episodios, 122 ocurrieron en el primer ciclo, 62 en el segundo, 32 en el tercero, 12 en el cuarto, 13 en el quinto, 11 en el sexto y el resto en los siguientes, con cifras muy pequeñas. En la Figura 1 se observa claramente cómo disminuyen estos episodios a

medida que se continúa con la ingestión de la droga. La proporción en la ocurrencia de estas metrorragias es igual para cada una de las distintas dosis.

En el Cuadro 4 se aprecia la ocurrencia de amenorrea secundaria. La mitad aproximadamente de los casos ocurrieron en mujeres que no estaban lactando.

Los siguientes síntomas fueron los que más comúnmente se presentaron con todas las dosis y se enumeran

\section{CUADRO 4}

OCURRENCIA DE AMENORREA SECUNDARIA EN MUJERES LACTANDO Y NO LACTANDO SEGUN LA DOSIS DIARIA DE INGESTION DE PASTILLAS

\begin{tabular}{|c|c|c|c|c|c|c|}
\hline \multirow[b]{2}{*}{ Dosis en Mg. } & \multirow[b]{2}{*}{ Pacientes } & \multicolumn{2}{|c|}{ Amenorrea } & \multirow[b]{2}{*}{ Total } & \multirow{2}{*}{\multicolumn{2}{|c|}{$\begin{array}{l}\% \text { de amenorrea } \\
\text { en no lactantes }\end{array}$}} \\
\hline & & Lactando & No lactando & & & \\
\hline 0,50 & 131 & 9 & 7 & 16 & & 44 \\
\hline 0,60 & 102 & 10 & 5 & 15 & & 33 \\
\hline 0,75 & 87 & 7 & 10 & 17 & & 59 \\
\hline 0,85 & 88 & 9 & 11 & 20 & & 55 \\
\hline TOTAL & 408 & 35 & 33 & 68 & & 48 \\
\hline
\end{tabular}


FIGURA $\mathrm{N}^{\circ}$ I

\section{OCURRENCIA DE METRORRAGIA SEGUN EL CICLO}

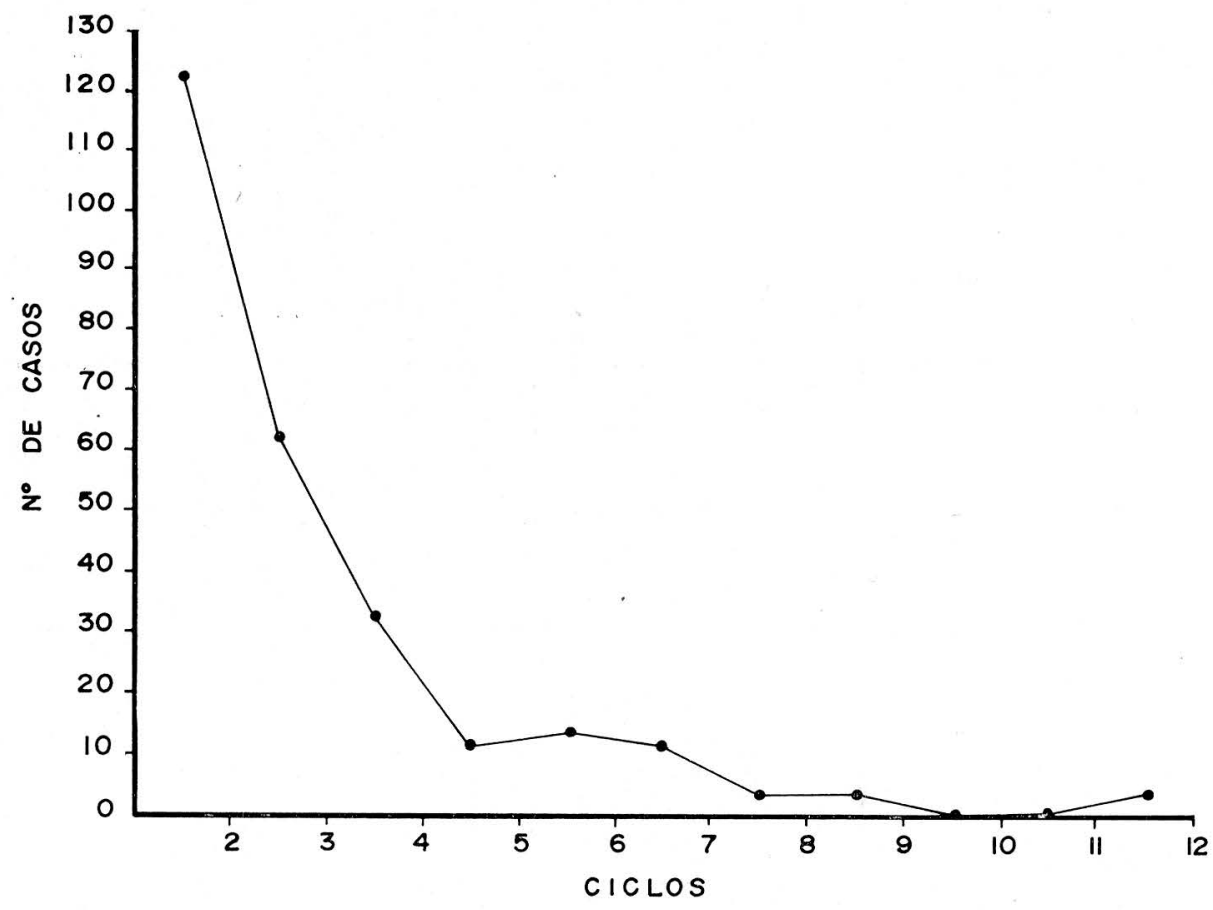

CUADRO 5

TASA (POR CIEN MUJERES) DE SUSPENSION DEFINITIVA DE LA INGESTION CONTINUA DE ACETATO DE CLORMADINONA, SEGUN LA CAUSA MEDICA Y LA DOSIS

\begin{tabular}{|c|c|c|c|c|c|}
\hline Causa de suspensión & & Dosi & en $\mathrm{Mg}$. & & Total \\
\hline & 0,50 & 0,60 & 0,75 & 0,85 & \\
\hline Metrorragia & 0,8 & 1,0 & 4,6 & 1,1 & 1,7 \\
\hline Oligomenorrea & & & & 1,1 & 0,2 \\
\hline Cloasma & & & & 1,1 & 0,2 \\
\hline Várices sintomáticas & & 1,0 & & & 0,2 \\
\hline Cefálea & & & 1,1 & 1,1 & 0,5 \\
\hline Pérdida de peso y nerviosismo & & 2,0 & & 1,1 & 0,7 \\
\hline Nerviosismo & & & 1,1 & 1,1 & 0,5 \\
\hline Náuseas y mareos & 0,8 & & & & 0,2 \\
\hline Pérdida de peso & & 2,0 & 1,1 & & 0,7 \\
\hline TOTAL & 1,5 & 5,9 & 8,0 & 6,8 & 5,1 \\
\hline NUMERO & 131 & 102 & 87 & 88 & 408 \\
\hline
\end{tabular}

en orden decreciente de importan- viosismo, cloasma, trastornos vasculacia: cefálea, disminución de la líbido, res y mareos. En general, todos teaumento de la misma, náuseas, ner- nían tendencia a ocurrir en los pri- 
meros meses de uso de la droga y se aminoraban en intensidad y frecuencia con el paso del tiempo.

Las causas de retiro las hemos dividido en médicas y no médicas. En el Cuadro 5 se observa que la mayoría de las razones médicas importantes para abandonar el método ocurren con las dosis de 0,75 y 0,85. Con la de 0,6 las razones son de menor imnortancia casi siempre, y con la de 0,5 se aducen solo en unos pocos casos.

En el Cuadro 6 se observan las causas no médicas de retiro, las cua-

\section{CUADRO 6}

TASA (POR CIEN MUJERES) DE SUSPENSION DEFINITIVA DE LA INGESTION CONTINUA DE ACETATO DE CLORMADINONA SEGUN LA CAU-

\section{SA NO MEDICA}

\begin{tabular}{lc} 
Causa & $\begin{array}{c}\text { Tasa por 100 } \\
\text { de suspensión }\end{array}$ \\
\hline Traslado a otro Municipio & 2,9 \\
Cambio a otra droga & 1,2 \\
Prohibición del esposo & 1,0 \\
Pérdida de seguimiento & 1,0 \\
No querer tomar pastillas & \\
diariamente & 0,5 \\
Motivos religiosos & 0,5 \\
Falta de especificación & 0,2 \\
en el calendario & 0,2 \\
Deseo de nuevo embarazo & 7,6 \\
TOTAL & \\
\hline
\end{tabular}

les son de distinto orden y no tienen influencia en la evaluación de la droga.

Finalmente, el Cuadro 7 muestra las omisiones que ocurrieron con el uso de las distintas dosis. Se observa que a pesar de llegar a 97 en total, solamente ocurrió un embarazo en los cuatro grupos, lo cual parece significar que el efecto anticonceptivo so manicieno por varios días cuando ocurre omisión de pastillas.

\section{Conclusiones}

1. El efecto anticonceptivo de las dosis de 0,5,0,6, 0,75 y 0,85 mg. de acetato de clormadinona no puede conocerse aún en esta investigación, por falta de un número adecuado de meses de uso. Hasta el momento, parece que fuera alto.

2. Los principales efectos adversos son: cefálea, trastornos de la líbido, náuseas y nerviosismo, y tienden a disminuir con el uso continuado de la droga.

3. La mitad de los ciclos mantiene una periodicidad normal, y ocurre un acortamiento en un $33 \%$ de los casos. Los episodios de hemorragia intermenstrual ocurren en la mayor parte de los casos en los dos primeros ciclos.

CUADRO 7

PASTILLAS OMITIDAS POR CICLO

\begin{tabular}{lccccc}
\hline Pastillas omitidas & Total mujeres & \multicolumn{3}{c}{ Dosis en $\mathbf{M g}}$. \\
\hline & & 0,50 & 0,60 & 0,75 & 0,85 \\
$1-3$ & 47 & 6 & $16(1)$ & 11 & 14 \\
$4-6$ & 27 & 3 & 10 & 5 & 9 \\
7 y más & 23 & 5 & 3 & 7 & 8 \\
TOTAL & 97 & 14 & 29 & 23 & 31 \\
\hline
\end{tabular}

(1) El único embarazo que se presentó en esta serie de mujeres, ocurrió en una del grupo que se señala y terminó en un aborto a los tres meses. 
4. La aceptabilidad del método es buena.

\section{Summary}

1. The contraceptive effect of chlormadinone acetate in the doses of 0.5 , $0.6,0.75$ and $0.85 \mathrm{mg}$. cannot be determined as yet from this investigation be cause of the lack of an adequate number of women-months of use of the different doses. So far it seems to be high.

2. The main untoward effects are: headache, disturbances of the libido, nausea, nervousness, and they tend to diminish with the continued use of the drug.

3. Half of the menstrual cycles maintain a normal rhythm and there is a shortening of them in 33\% of the cases. Intermenstrual bleeding occurs mainly in the first two cycles.

4. The method has a good acceptance.

\section{BIBLIOGRAFIA}

1 MARTINEZ-MANAUTOU, J. \& RUDEL, H. W.: Low dose continuous progestogen therapy. Fertility and Sterility, Stockholm, Sweden. 22 June 1966. Excerpta Medica. Cit. por Martínez-Manautou (2).

2 MARTINEZ-MANAUTOU, J., GINER-VELASQUEZ, J., CORTES-GALLEGOS, $\vee$., AZNAR, R., ROJAS, B., GUTIERREZ-NAJAR, A., RUDEL, H. W.: Daily progestogen for contraception: a clinical study, Brit. Med. J.: 2: $730-732$ Presented at the Fifth World Congress en (Jun. 17), 1967.

3 ZAÑARTU, J., RODRIGUEZ-MOORE, G., PUPKIN, M., SALAS, O. Y GUERRERO, R.: Antifertilyity effect of continuous low dosage oral progestogen therapy. Brit. Med. J., 2: 263266 (May 4), 1968.

\section{Agradecimientos :}

Presentamos nuestros sinceros agradecimientos al Sr. Marcelo Huerta, profesor de Estadística de la Escueia de Salud Pública, por su ayuda para la elaboración de los cuadros y gráfica de este trabaịo. 